\title{
Distinctive Responses in the Medial Amygdala to Same- Species and Different-Species Pheromones
}

\author{
Michael Meredith and Jenne M. Westberry \\ Program in Neuroscience and Department Biological Science, Florida State University, Tallahassee, Florida 32306-4340
}

\begin{abstract}
Chemosignals related to reproductive and social status (pheromones) carry messages between opposite-sex and same-sex individuals in many species. Each individual must distinguish signals relevant to its own social behavior with conspecifics from signals used by other (heterospecific) species relevant to their social behavior. In male hamsters, the medial amygdala responded in a categorically different way to conspecific stimuli (socially relevant) and heterospecific stimuli (not socially relevant but serving similar purposes for other species), and may play an important role in this decision. Immediate-early gene responses to conspecific chemosignals and heterospecific chemosignals were characteristically different. The categorical responses, generated by chemosensory input from the vomeronasal organ and (probably) GABA inhibition within the amygdala, were not apparent at more peripheral sensory levels. This is the first evidence for an important role of the amygdala, a limbic structure known to be involved in social and emotional behavior, in discrimination of species specificity in chemosignals.
\end{abstract}

Key words: amygdala; vomeronasal; hamster; medial nucleus; pheromone; accessory olfactory bulb

\section{Introduction}

Chemical signals are an important communication medium for most mammals, and have significance even in humans (Jacob et al., 2002). Signals with preprogrammed meaning (pheromones) [see Meredith (2001) for definition] carry information essential for reproductive and social behavior between members of the same species (Wysocki and Meredith, 1987). Animals discriminate between chemosignals from their own species (conspecific) and those from other species (heterospecific) (Murphy, 1980; Johnston and Brenner, 1982). Here we show that the vomeronasal chemosensory system responds to both types of chemosignals, and that its central projections discriminate between them.

Vomeronasal information passes via the accessory olfactory bulb (AOB) to the "vomeronasal amygdala," principally the medial amygdala. Main olfactory information passes via the main olfactory bulb to the piriform cortex and "olfactory amygdala," principally the anterior cortical amygdala, but with onward connections to the medial amygdala (Meredith, 1998). Increased immediate-early gene (IEG) expression, a marker of neural activity (Morgan and Curran, 1991), indicates that the medial amygdala in male hamsters is more strongly activated during mating or by female chemosensory stimuli than are the main olfactory areas (Kollack-Walker and Newman, 1997; Meredith, 1998).

Hamsters actively investigate chemosignals that are used for social communication by other species, including those of mice

Received March 26, 2004; revised May 4, 2004; accepted May 5, 2004.

This work was supported by National Institute on Deafness and Other Communication Disorders Grants DC 005813 and T32 DC00044 (M.M.) and fellowship F31 DC05725 (J.M.W.). We thank Dr. Christopher M. Nolte for permission to include Figure $2 b$ from his unpublished work.

Correspondence should be addressed to Dr. Michael Meredith, Department Biological Science (4340), Florida State University, Tallahassee, FL 32306-4340. E-mail: mmered@neuro.fsu.edu.

DOI:10.1523/JNEUROSCI.1139-04.2004

Copyright $\odot 2004$ Society for Neuroscience $\quad$ 0270-6474/04/245719-07\$15.00/0 (potential competitors) and cats (potential predators). We exposed male hamsters to various chemosensory stimuli (delivered on cotton swabs) from conspecific and heterospecific females and males. We measured expression of IEG proteins, Fos, and Fos-related antigens (FRAs) in brain sections to estimate neural activation in central chemosensory pathways. Conspecific stimuli included female hamster vaginal fluid (HVF), which is used by females to attract males, and flank gland secretion (FGS) from male and female hamsters (mFGS and fFGS, respectively), which is used by both sexes to indicate their presence and social status (as well as their individual identity) (Johnston and Brenner, 1982). The heterospecific stimuli included male mouse urine (mMU) and female mouse urine (fMU), which are used for both sexual- and social-status signaling (Bronson, 1979), and male cat urine $(\mathrm{mCU})$, which is used in marking territory (Verberne, 1976). To reduce the probability that our results might depend on learned responses or animals whose neuronal connectivity had been modulated by experience, we used sexually naive males with no exposure to females since weaning. They were individually housed in single-species rooms, had no previous exposure to chemosignals of the other species, and never encountered the specific individuals of their own species whose chemosignals were used in the experiments.

\section{Materials and Methods}

Animals. Sexually naive adult male hamsters (Mesocricetus auratus) were maintained on a 14/10 hr light/dark photoperiod and given food and water ad libitum. Animal procedures were approved by the Florida State University Institutional Animal Care and Use Committee.

Stimuli. HVF was collected with a small smooth spatula. Male and female FGS was collected by pinching skin around the gland and rubbing a swab up and down 10 times. FGS sources were different for each exposure, with three donors per swab. HVF and fFGS were collected from naturally cycling females on the day of behavioral estrus. Male and female 
mouse urine were collected from several male or female mice placed in a metabolic cage for three to five nights. Female sources were naturally cycling, including all estrous stages. Fresh urine from two neutered male house cats was collected by lining a litter box with clean absorbent paper, which was centrifuged to collect urine. All stimuli except FGS were diluted 1:10 with distilled water. Peppermint extract (McCormick, Hunt Valley, MD) was diluted 1:100 with distilled water.

Stimulus presentation. Stimuli on cotton swabs, or clean swabs, were delivered every $3 \mathrm{~min}$ for a total of $15 \mathrm{~min}$. The hamster was returned to its home cage and perfused after an additional $30 \mathrm{~min}$. In experiments involving olfactory lesions, there were also mating and food discrimination tests $10 \mathrm{~min}$ before perfusion to measure the animal's "readiness to mate" and general anosmia, respectively (Meredith, 1986; FernandezFewell and Meredith, 1998).

Olfactory lesions. Main olfactory lesions were made by intranasal infusion of $0.17 \mathrm{M}$ zinc sulfate (ZS). Animals were anesthetized with halothane and placed prone and head-down on an inclined surface while 0.1 $\mathrm{ml}$ of solution was injected per nostril (Fernandez-Fewell and Meredith, 1998). The 25 ga injection needle was blunted, laterally flattened, and bent dorsally to deliver a fine spray to the nasal area. Control animals received saline and all were allowed $5 \mathrm{~d}$ to recover.

Immunocytochemistry. Forty-five minutes after initial stimulus exposure, animals were perfused with $4 \%$ paraformaldehyde. Free-floating 40 $\mu \mathrm{m}$ coronal sections (cut on a freezing microtome) were incubated in rabbit anti-Fos (SC52; 1:50,000; Santa Cruz Biotechnology, Santa Cruz, CA) or anti-FRAs (SC253; 1:25,000; Santa Cruz Biotechnology) primary antiserum for 16-24 hr. Sections were washed in $0.1 \mathrm{M}$ PBS and incubated in biotinylated goat anti-rabbit secondary antiserum (1:400; Vector Laboratories, Burlingame, $\mathrm{CA}$ ) for $1 \mathrm{hr}$, followed by $\mathrm{ABC}$ and $\mathrm{DAB}$ (Vector Laboratories). For Fos and FRAs expression, we averaged counts of immunoreactive (IR) nuclei in two sections per anatomical nucleus; in anterior medial amygdala (MeA), posterior medial amygdala $(\mathrm{MeP})$, or intercalated nucleus (ICN); or in the three $40 \mu \mathrm{m}$ sagittal sections from the middle of the AOB. Separate counts were made in mitral and granule cell layers for rostral and caudal AOB, as determined by landmarks in sections stained for G-proteins ( $\mathrm{G} \alpha \mathrm{i} 2$ in rostral $\mathrm{AOB}$ and $\mathrm{G} \alpha \mathrm{o}$ in caudal $\mathrm{AOB})$. Here we show fluorescent staining (Texas Red) for $\mathrm{G} \alpha \mathrm{O}$ (1:500; Santa Cruz Biotechnology).

Statistics. Comparisons of immediate-early gene expression elicited by various stimuli in the $\mathrm{MeA}$ and $\mathrm{MeP}$ or in the rostral and caudal $\mathrm{AOB}$ were made by two-way repeated-measures (RM) ANOVAs with the following factors: area (MeA, MeP or rostral $\mathrm{AOB}$, caudal $\mathrm{AOB}$ ) versus exposure (various chemosensory stimuli). In separate ANOVAs, the chemosensory stimuli were either ungrouped or were grouped as conspecific, heterospecific, and control. RM analysis was used because the different brain areas were from the same set of animals. For the artificial stimulation experiments, two-way RM ANOVAs were used to compare the ipsilateral and contralateral sides of stimulated animals as well as the equivalent sides of unstimulated animals. For the experiments with intranasal zinc sulfate or saline, IEG expression was analyzed within each brain area $[\mathrm{MeA}, \mathrm{MeP}, \mathrm{ICN}$, anterior cortical nucleus (ACN), and piriform cortex (PC)] by separate two-way repeated-measures ANOVAs with the following factors: treatment (ZS or saline) versus exposure (various stimuli, considered independently).

Vomeronasal organ stimulation. The vomeronasal organ (VNO) was stimulated in awake animals using Teflon-insulated silver-wire electrodes, previously implanted under anesthesia, with electrode tips inside the VNO capsule (Meredith and Fewell, 2001). Three days after surgery, animals were placed in a clean plastic cage and connected via flexible wires to a constant-current stimulator. Four control animals with electrodes implanted were not stimulated. Six other animals were stimulated via the VNO electrodes at $120-175 \mu \mathrm{A}$ with trains of 10 pulses at $10-20$ $\mathrm{Hz}$ every $2 \mathrm{sec}$, for $20 \mathrm{~min}$, providing bursts of activity in VNO sensory neurons or their axons; this mimicked the timing of stimulation provided by the vascular pumping mechanism that draws stimuli into the VNO (Meredith, 1994). Current levels used were at least $150 \mu \mathrm{A}$ below those producing motor reflex movements in pilot experiments and did not produce any signs of discomfort. After $20 \mathrm{~min}$ of stimulation (or without stimulation for controls) and an additional 25 min for protein expression, animals were perfused.

Metabotropic glutamate receptor-2 agonist. Males were implanted with 28 ga guide tubes rostral to the $\mathrm{AOB}$, to avoid $\mathrm{AOB}$ damage but allow injected solutions to infuse into the AOB. Metabotropic glutamate receptor-2 (mGluR2) expression is low in the main olfactory bulb, allowing selective activation of the AOB. Seven days after surgery, awake animals were injected with $1 \mu \mathrm{l}$ of $\left(2 S, 1^{\prime} S, 2^{\prime} S\right)$-2-(carboxycyclopropyl)glycine (LCCG-I; Tocris Cookson, Ellisville, MO) at $4 \mu \mathrm{g} / \mathrm{ml}$ (or with saline for controls) through a 33 ga cannula fitted to the guide tube on one side. After 90 min, animals were perfused.

\section{Results \\ Categorical response to conspecific stimuli in medial amygdala}

We found increased Fos protein expression in the AOB and the medial amygdala with both heterospecific and conspecific stimuli, indicating that both activate the vomeronasal sensory pathway. The amygdala response showed a categorical discrimination that was not apparent at the level of the AOB. Conspecific stimuli, whether from males or females, significantly activated the medial amygdala, both MeA and $\mathrm{MeP}(p<0.01)$ (Fig. $1 a, b)$. Heterospecific stimuli failed to activate the MeP even when they activated the MeA equally or more strongly than conspecific stimuli (Fig. $1 a, c)$. This pattern was the same when measured by Fos expression (Fig. 1) or FRAs expression (see Fig. 4, saline) and was maintained consistently for the multiple examples of heterospecific and conspecific stimuli tested. In the posterior medial amygdala, responses to conspecific stimuli were significantly different from responses to heterospecific stimuli or to control stimuli (both, $p<0.001)$. Responses to heterospecific stimuli in the $\mathrm{MeP}$ were not significantly different from controls $(p=0.272)$. In MeA, responses to both conspecific and heterospecific stimuli were different from controls (both, $p<0.001$ ) but not different from each other $(p=0.596)$. Statistical probabilities are from Tukey post hoc tests after a two-way repeated-measures ANOVA with the following factors: area (MeA, MeP) and exposure (the stimuli shown in Fig. $1 a-c$ grouped as conspecific, heterospecific, or control). When stimuli were not grouped, responses to all individual conspecific stimuli were significantly greater than to clean-swab control stimuli in the MeA and MeP. Responses to heterospecific stimuli were significantly greater than controls only in the MeA (Fig. $1 a-c$, asterisks) $(p<0.01)$.

Animals investigated all clean and scented swabs with no significant differences in investigation time between scented swabs (Table 1, intact group). Clean swabs elicited approximately onethird the investigation time of scented swabs. Peppermint was investigated as much as the animal-source stimuli but produced no significant Fos expression in the MeA, MeP, or AOB. The numbers of animals exposed to each stimulus (Table 1) also apply to the graphs of the immediate-early gene response.

Two lines of evidence suggest that the categorical response in the MeP is a property of amygdala function. First, patterns of activation in the AOB, the proximal afferent relay for the amygdala, did not show the same categorization. Rostral and caudal AOB compartments receive input from different sets of vomeronasal sensory neurons, expressing different families of vomeronasal receptor (VR) genes and different G-proteins (Dulac and Torello, 2003) (G-protein distribution confirmed for the hamster in Fig. $1 g$ ). Relative activation of these compartments indicates differential activation of sets of sensory neurons. Figure $1 d-h$ shows counts of Fos-IR nuclei in the mitral layer. Both the conspecific and heterospecific stimuli significantly activated the $\mathrm{AOB}$ $(p<0.001)$, and there was a marginally significant difference 

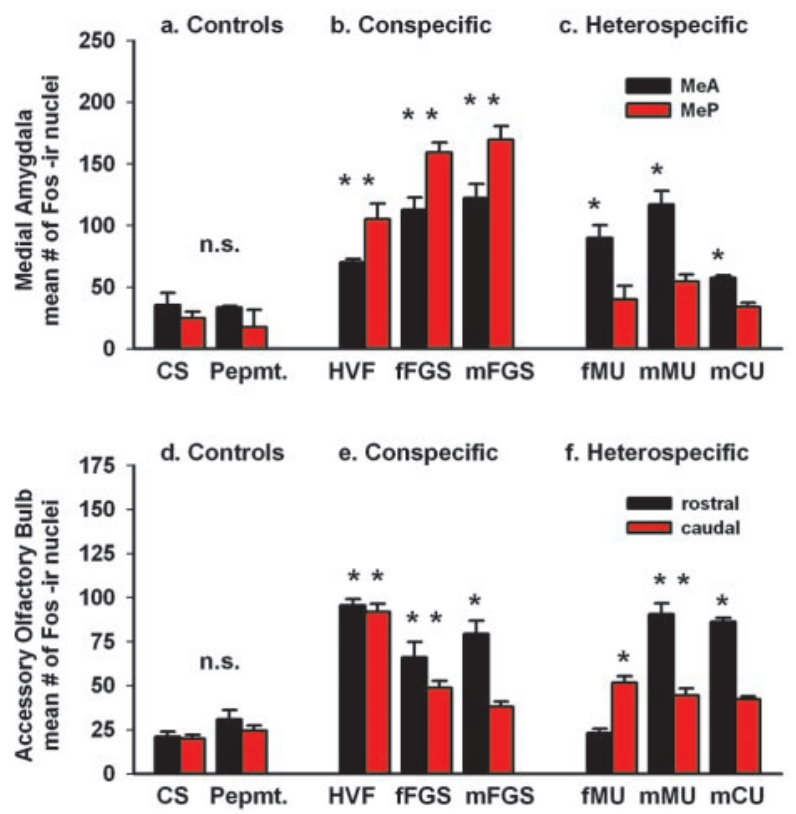

g. Go -ir in caudal $A O B$

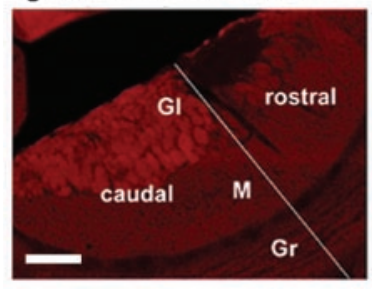

h. HVF stimulated Fos in AOB

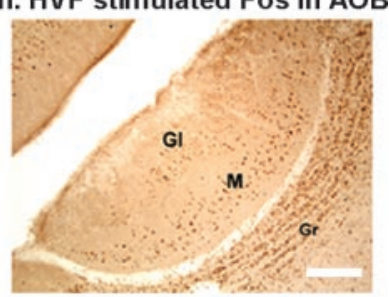

Figure 1. Categorical response to conspecific versus heterospecific chemosignals in the amygdala but not in the AOB. Compared with controls ( $a$ ), conspecific (socially relevant) stimuli (b) elicited significant Fos activation in both the MeA and MeP $(p<0.01)$. Heterospecific (socially nonrelevant) stimuli $(c)$ significantly activated the MeA $(p<0.01)$, but this category failed to activate the MeP ( $p=0.272$; probabilities were obtained from post hoc tests after two-way RM ANOVA on grouped data). Asterisks show differences $(p<0.01)$ between responses to individual stimuli and responses to unstimulated clean-swab (CS) controls (two-way RM ANOVA on individual data). n.s., Nonsignificant. $d-f$, Mitral cells of the rostral and caudal $A O B$ show no categorical response difference between conspecific and heterospecific stimuli. The distribution of Fos expression to the rostral and caudal $A O B$ was not consistent within the conspecific or the heterospecific groups of stimuli. $g$, Go-protein immunoreactivity delimits the caudal AOB glomerular layer. The diagonal line divides rostral from caudal AOB. $h$, Mitral and granule cells show the same pattern of response (to HVF here). M, Mitral layer; Gl, glomerular layer; Gr, granule layer; Pepmt., peppermint. Scale bars: $g, h, 50 \mu \mathrm{m}$.

between responses to conspecific and heterospecific stimuli in the AOB as a whole $(p<0.04)$ when stimuli were grouped as conspecific, heterospecific, or control. Both types of stimuli significantly activated both rostral and caudal subdivisions (all $p<$ 0.014 ), but responses in the two subdivisions were not significantly different from each other $(p=0.086$, rostral; $p=0.207$, caudal). These probabilities are from Tukey post hoc tests after a two-way RM ANOVA with the following factors: area (rostral $\mathrm{AOB}$, caudal $\mathrm{AOB}$ ) and exposure (stimuli grouped as above). However, the grouped data do not capture the consistent differences between responses to individual stimuli within the groups. In a second two-way RM ANOVA with stimuli ungrouped, there was a significant main effect of stimulus exposure $(p<0.01)$, indicating that stimuli differed in effectiveness. All of the individual stimuli activated rostral AOB or caudal AOB, or both $(p<$ 0.01 ) (Fig. $1 d-f$, asterisks), and all stimuli except HVF, peppermint, and clean swabs activated one subdivision more than the other. However, there was no consistency within the conspecific or heterospecific groups. All of the conspecific stimuli activated the rostral AOB, but of those stimuli, only HVF and fFGS activated the caudal AOB. Among heterospecific stimuli, male mouse urine activated both the rostral and caudal $\mathrm{AOB}$, but male cat urine activated only the rostral $\mathrm{AOB}$ and female mouse urine activated only the caudal AOB. Even when judged by the simple criterion of relative activation of the rostral and caudal AOB, the AOB responses to conspecific and heterospecific signals do not fall into the same groups that they do in the amygdala. Thus, the category of stimuli that activates the $\mathrm{MeP}$ does not do so because those stimuli simply activate identical sets of sensory neurons.

The second line of evidence indicating that the categorical response arises in the amygdala comes from artificial stimulation of the vomeronasal system. Unexpectedly, electrical stimulation of the VNO in awake animals via implanted electrodes activated less of the downstream circuit than did conspecific chemosensory stimulation. Instead, it strongly activated the MeA $(p<0.01)$ but not the MeP, which is the same pattern observed for heterospecific chemosensory stimuli. This same result for electrical stimulation is seen regardless of whether activation is measured by Fos expression (Meredith and Fewell, 2001) or FRAs expression (Fig. $2 a$ ). We also injected LCCG-I, a mGluR2 agonist, into the accessory olfactory bulb (Nolte and Meredith, 1999) to activate the system artificially. The drug activates the system by reducing feedback inhibition of AOB output neurons (Hayashi et al., 1993). Unlike the electrical stimulation, which was delivered in discrete bursts to mimic natural stimulation (Meredith, 1994), the drug effect rises and falls slowly. Nevertheless, LCCG-I stimulation of $\mathrm{AOB}$ output also significantly activated Fos expression in the MeA $(p<0.01)$ but not in the MeP (Fig. $2 b)$. We attribute the similar outcome of these experiments to the fact that both methods produce nonspecific activation of $\mathrm{AOB}$ output neurons.

\section{Activation of intercalated (GABA) neurons by heterospecific stimuli}

We have preliminary evidence that failure to activate the $\mathrm{MeP}$ is an active process involving inhibition. Heterospecific stimuli failed to activate the MeP but did increase activation in the ICN, which has $\sim 80 \%$ GABA-containing neurons (Nitecka and BenAri, 1987). The hamster amygdala also has a high level of GABA immunoreactivity in the ICN, as shown in Figure $3 a$ (arrowheads) by GABA-IR fluorescence in the caudal group of ICN cells (ICNc) that lies adjacent to MeP. For these experiments, stimuli were delivered as before, but we used the FRAs-IEG system (Lerant et al., 2001) to register neural activation in the ICN, which does not express Fos in our experiments. FRAs expression correlates with the amount of stimulation and the level of Fos expression in areas that express both Fos and FRA (e.g., AOB, MeA, and $\mathrm{MeP}$ ) under a range of stimulus conditions, increasing our confidence that both indicate neuronal activation.

Figure $3 b$ shows FRAs expression in low-magnification views of the MeA, MeP, and ICNc from males exposed to clean swabs or to conspecific or heterospecific stimuli. The data for the groups of animals exposed to these and additional stimuli are plotted in Figure $3 c$. The reciprocal relationship between activation in the ICNc and in the adjacent MeP (Fig. 1c) for heterospecific stimuli suggests inhibition of the MeP by the ICNc. This relationship holds for at least one conspecific stimulus, HVF, which strongly activated the MeP and depressed activation in the ICNc (Figs. $3 b, c, 4 e$ ). In preliminary experiments (data not shown), HVF significantly suppressed activation of GABA-IR cells in the ICNc. Thus, the discriminant activation in the MeP by stimuli from 
Table 1. Investigation time (in seconds) for different animal groups and different stimuli

\begin{tabular}{|c|c|c|c|c|c|c|c|c|}
\hline \multicolumn{4}{|c|}{ Controls } & \multicolumn{3}{|l|}{ Conspecific } & \multicolumn{2}{|l|}{ Heterospecific } \\
\hline & CS & Peppermint & HVF & fFGS & $\mathrm{mFGS}$ & fMU & $\mathrm{mMU}$ & $\mathrm{mCU}$ \\
\hline Intac & $12 \pm 3.01(n=6)$ & $28 \pm 1.01(n=6)$ & $35 \pm 1.0(n=6)$ & $26 \pm 2.9(n=6)$ & $24 \pm 4.6(n=6)$ & $33 \pm 3.8(n=6)$ & $36 \pm 3.9(n=6)$ & $24 \pm 8.6(n=4)$ \\
\hline Salin & e $14 \pm 2.91(n=6)$ & Not tested & $30 \pm 2.9(n=5)$ & $38 \pm 3.86(n=5)$ & $32 \pm 3.91(n=5)$ & $34 \pm 5.11(n=5)$ & $42 \pm 4.13(n=5)$ & $33 \pm 4.63(n=4)$ \\
\hline OLFX & $8 \pm 4.84(n=7)$ & Not tested & $28 \pm 5.21(n=6)$ & $27 \pm 3.31(n=5)$ & $33 \pm 7.30(n=6)$ & $29 \pm 2.36(n=6)$ & $34 \pm 9.89(n=6)$ & $26 \pm 4.98(n=6)$ \\
\hline
\end{tabular}

Animal groups are not different. For each group, the clean swab (CS) is different from all other stimuli. Scented swabs are not significantly different from each other [two-way ANOVA comparing group (intact, saline, 0LFX) and exposure (various stimuli)].

\section{a. VNO electrical stimulation}

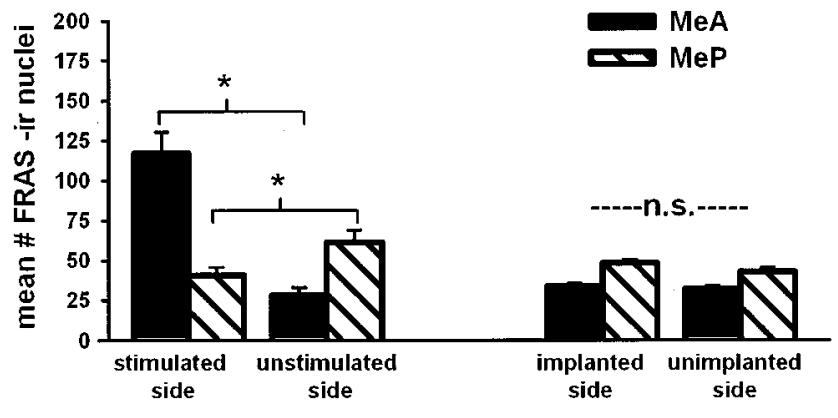

Electical stimulation

Implanted controls

\section{b. Unilateral Cannula Delivery of LCCG}

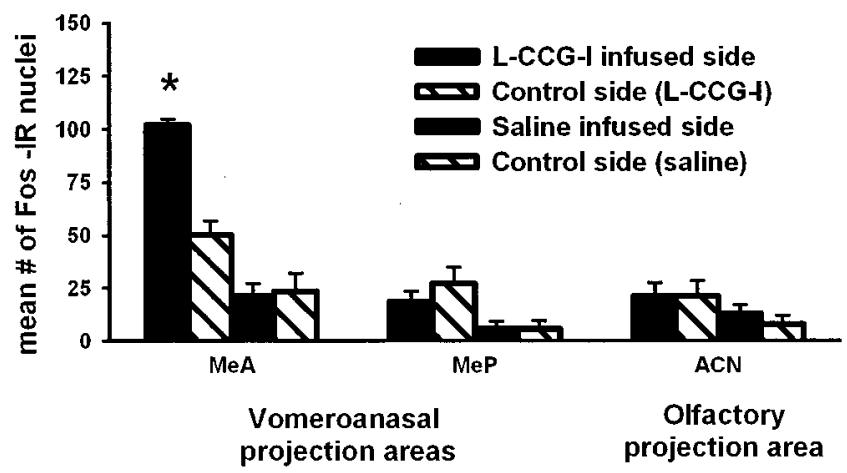

Figure 2. Artificial stimuli, resulting in abnormal spatiotemporal patterns of input to the amygdala, failed to activate the MeP and are categorized with heterospecific stimuli. $a$, FRAs expression was significantly greater $\left({ }^{*} p<0.01\right)$ in the MeA ipsilateral to electrical stimulation of one vomeronasal organ than on the unstimulated side, or in electrode-implanted, unstimulated controls [two-way RM ANOVA with the following factors: side (ipsilateral, contralateral) and treatment (stimulated, unstimulated) with Tukey post hoc tests; n.s., nonsignificant]. The MeP was not activated; in fact, FRAs expression was significantly less $\left({ }^{*} p<0.01\right)$ on the stimulated side (ANOVA, as above). $b$, The mGluR2 agonist LCCG-I disinhibited (increased) AOB output when infused unilaterally into the $A O B$, initiating significantly greater infusion-side Fos expression in the MeA ${ }^{*} p<0.01$ ) than on the control side or on either side of saline-infused animals. LCCG-I infusion produced no significant activation in the MeP or in the ACN of the olfactory amygdala (ANOVA, as above for each area).

different species may depend on selective GABA inhibition from the ICNc during heterospecific stimulation. Artificial stimulation of the vomeronasal pathway by unilateral electrical stimulation of the VNO also significantly activated the ICNc on the ipsilateral side (ANOVA; $p<0.01$ ) (Fig. $3 c$, right) while activating the MeA but not the MeP. The common outcome of artificial stimulation and heterospecific stimulation may share a common mechanism if both generate spatiotemporal patterns of input to the amygdala that fall outside those expected for conspecific stimuli. a.

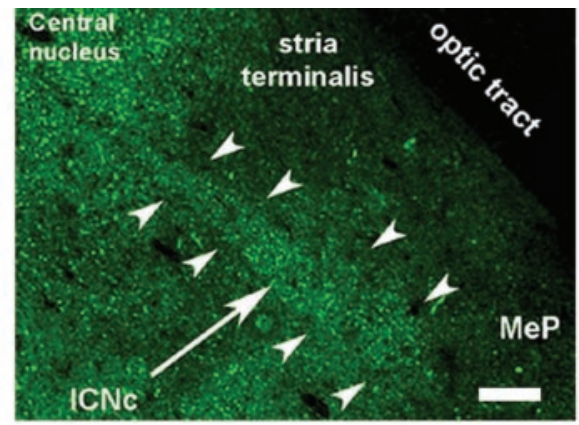

b.

Hamster

Male

$\begin{array}{ccc}\begin{array}{c}\text { Clean swab } \\ \text { (control) }\end{array} & \begin{array}{c}\text { vaginal fluid } \\ \text { (conspecific) }\end{array} & \begin{array}{c}\text { mouse urine } \\ \text { (heterospecfic) }\end{array}\end{array}$

Ant.

medial

amygdala

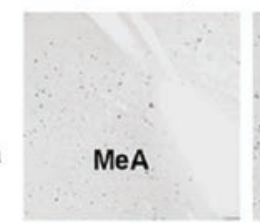

(heterospecfic)

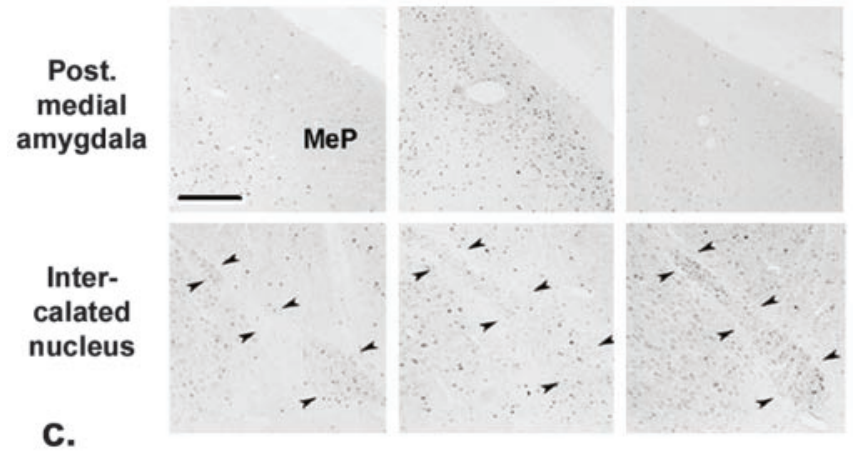

C.

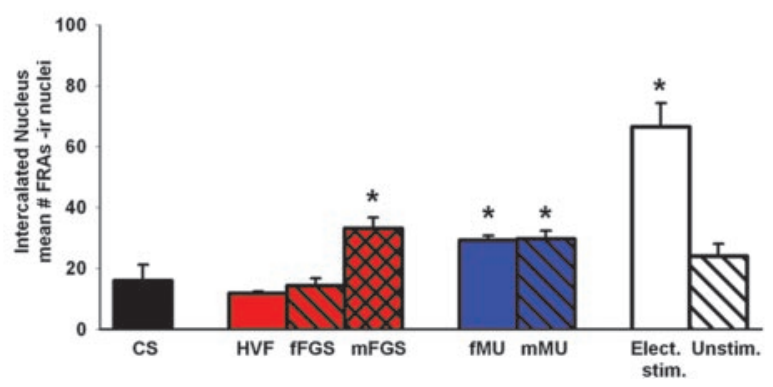

Figure 3. GABA-IR cells in the ICNc (outlined by arrowheads in $a$ and $b$ ) may inhibit the MeP. $a$, GABA immunofluorescence (green) in the ICNc (arrow). b, A conspecific stimulus (HVF) activated the MeP but not the ICNc. A heterospecific stimulus (mMU) failed to activate the MeP but significantly activated the ICNc. Ant., Anterior; Post., posterior. c, Group data. All heterospecific stimuli activated the ICNc [ ${ }^{*} p<0.01$ vs clean-swab (CS) controls; post hoc tests after one-way ANOVA]. fFGS had no effect on the ICNc; mFGS significantly activated the ICNc (see Discussion) but also significantly activated the MeP (Fig. 1b). c, Right, Electrical stimulation (Elect. stim.) of the vomeronasal organ significantly activated the ICNC on the stimulated side $\left({ }^{*} p<0.01\right.$ vs unstimulated side or control; ANOVA as for Fig. 2a) and failed to activate the MeP (Fig. 2a). Unstim., Unstimulated. Scale bars: $a, b, 50 \mu \mathrm{m}$. 
a.

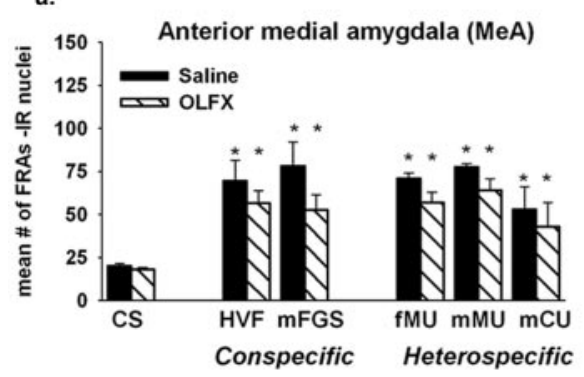

b.

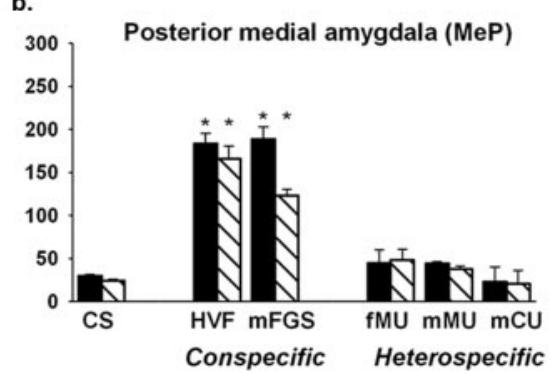

c.

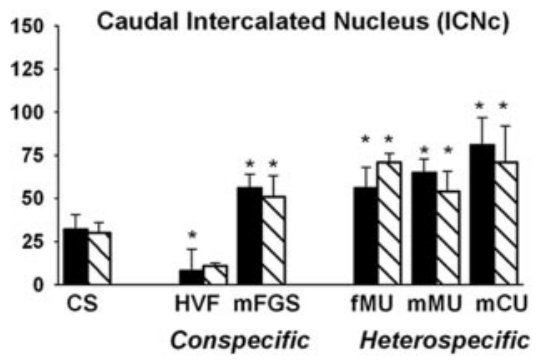

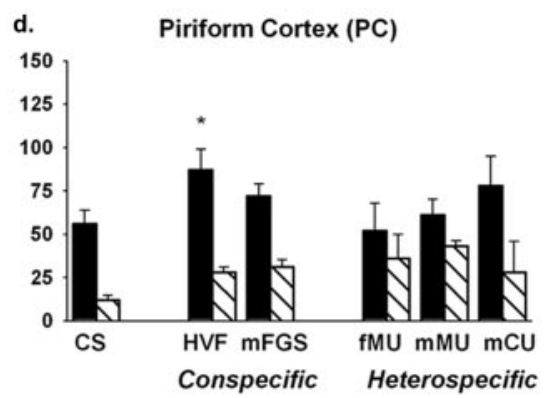

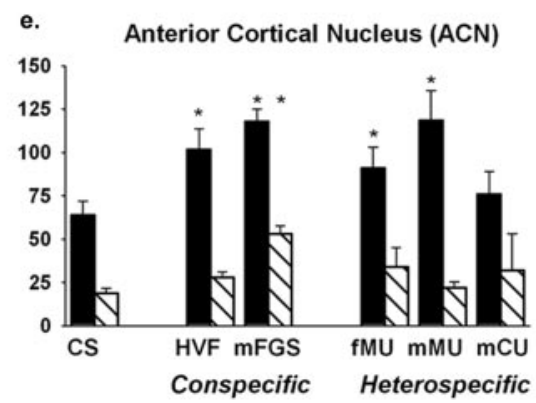

Figure 4. Normal main olfactory input is not necessary for categorical responses in the medial amygdala. After intranasal zinc sulfate treatment (OLFX), responses were not different from saline-infused controls. $a, b$, Conspecific stimuli still significantly activated FRAs expression in the MeA and MeP. Heterospecific stimuli still failed to activate the MeP. c, Heterospecific stimuli (and $\mathrm{mFGS}$ ) still significantly activated the ICNc in OLFX males. HVF significantly suppressed FRAs in the ICNc of saline-treated controls (asterisk above the HVF bars; $p<0.03$ ). $d, e, 0 \mathrm{LFX}$ animals had no significant FRAs activation in the main olfactory areas: $\mathrm{PC}$ and ACN (except by mFGS). Asterisks indicate a significant response compared with clean-swab (CS) controls ( $p<0.01 ; p$ ost hoc tests after two-way ANOVA for each brain area; see Results).

Main olfactory input is not necessary for categorical response in the medial amygdala

Artificial stimulation was restricted to the vomeronasal system, but chemosensory stimuli could also stimulate the main olfactory system, which has indirect access to the medial amygdala (Coolen and Wood, 1998). We tested whether main olfactory input is necessary for the categorical amygdala response by disabling main olfactory input (OLFX), using intranasal ZS infusion (Fernandez-Fewell and Meredith, 1998; Meredith and O'Connell, 1988). Comparing OLFX males and control males infused with saline, we found no essential differences in the characteristic patterns of FRAs expression in the anterior and posterior medial amygdala elicited by conspecific and heterospecific stimuli (Fig. 4a,b). Neither area showed a statistical main effect of ZS treatment in a two-way ANOVA for each area with the following factors: treatment (saline, ZS) and exposure (the various stimuli). There was a small decrease in FRAs expression in the $\mathrm{MeA}$, reflecting the small contribution from the main olfactory bulb (Lehman and Winans, 1982) and a small (nonsignificant) reduction in response to $\mathrm{mFGS}$. All heterospecific stimuli still activated the ICNc (Fig. 4c). In contrast, ZS treatment significantly reduced FRAs activation in the main olfactory brain regions, the anterior PC and the ACN of the olfactory amygdala (Fig. $4 d, e$ ), with a significant main effect of treatment in both of these areas (two-way ANOVA in each area, as above; $p<0.01$ ). All ZS-treated animals included here failed a food-odor discrimination test but did mate, a hallmark of olfactory loss in naive male hamsters (Fernandez-Fewell and Meredith, 1998). Salinetreated animals spent $90 \%$ of their time in the $3 \mathrm{~min}$ food-odor discrimination test sniffing and biting at the food port, significantly $(p<0.05)$ more than chance level (50\%) and significantly $(p<0.01)$ more than ZS-treated animals (36\%; not significantly different from chance; $t$ test). All males, both OLFX and control, achieved five or more intromissions in the 5 min mating test, a normal performance for naive animals (Fernandez-Fewell and Meredith, 1998). Both the food test and mating test were given immediately before the animals were killed so that the chemosensory stimulation involved in these tests would not contribute to observable FRAs protein expression.

\section{Discussion}

We show here, for the first time, that the medial amygdala in male hamsters responds categorically differently to conspecific and heterospecific chemosensory stimulation. The MeP is unresponsive to heterospecific stimuli and appears to be suppressed, possibly by the immediately adjacent GABA-rich ICN, which was activated by all stimuli that suppressed the MeP. The main olfactory system has connections with the medial amygdala but was not necessary for the categorization of responses there. The vomeronasal system was activated by both categories, but the accessory olfactory bulb response did not distinguish them categorically. We believe that the amygdala response, more centrally, reflects a higher level of analysis where, perhaps, social relevance is extracted.

The stimuli used in these experiments were unpurified natural products containing many extraneous chemicals that are different for different species, in addition to pheromones used for communication. These additional chemicals did not seem to affect the categorization of the stimuli by the amygdala. Pheromones are intraspecific chemical signals potentially of mutual benefit for sender and receiver (Meredith, 2001). Many, although not all (Johnston, 1998), are effective via the vomeronasal system, as appears to be the case for amygdala activation by our stimuli. Recent evidence suggests that vomeronasal sensory neurons may be narrowly tuned to specific molecules (Leinders-Zufall et al., 2000), unlike the main olfactory system, so why would one species respond to another's pheromones? Foreign pheromones would be unlikely to carry mutually beneficial information for 
another species, but they may have common chemical components. Hamsters probably also share a similar repertoire of functional VR genes with other rodents, as is the case for mice and rats (Dulac and Torello, 2003). Common chemicals would activate common receptors but could be components of different blends, each blend a pheromone only for one of the species. It is also possible that the VNO may have receptors (specialist or generalist) for nonpheromone substances. For example, snakes use the VNO to detect predators and prey (Halpern and MartinezMarcos, 2003).

AOB mitral cells respond differentially to stimuli from conspecific individuals according to gender and strain (Luo et al., 2003), presumably reflecting stimulation by different chemicals, of vomeronasal sensory neurons expressing different VR receptors (Del Punta et al., 2002). Differing spatiotemporal patterns of response in $\mathrm{AOB}$ mitral cells here result in categorically different responses to conspecific and heterospecific signals in the posterior medial amygdala, possibly by discrimination of patterns incident to the MeA. In contrast, artificial stimulation of the vomeronasal organ or $\mathrm{AOB}$ activates much or all of the $\mathrm{AOB}$ input to the amygdala, but nonspecifically and synchronously, a pattern unlikely to resemble any natural chemosensory message. This "white-noise" input to the amygdala may fail to activate circuits there that normally select behaviorally relevant patterns of input or may engage circuits that reject nonrelevant patterns of input. The common outcome of heterospecific and artificial stimulation may reflect a rejection of inputs that do not match expectations for conspecific stimuli. This would be consistent with our argument that discrimination of different classes of stimuli may be based on spatiotemporal patterns of input to the MeA. Whether information on heterospecific stimuli is discarded or analyzed elsewhere is unclear. However, the investigation times for heterospecific stimuli are little changed in OLFX animals and are significantly different from investigation times for clean swabs, suggesting that vomeronasally derived information is available to modify behavior, possibly via activation of the MeA. Heterospecific and artificial stimulation always activated the $\mathrm{MeA}$ and ICNc but not the MeP, and conspecific stimulation always activated the MeA and MeP. One conspecific stimulus, mFGS, also activated the ICNc (as well as the MeA and MeP), but we do not know which ICN cells are activated. We are currently investigating the cell phenotype and connections of hamster ICN neurons.

Although all of the heterospecific stimuli and none of the conspecific stimuli are urine, the categorical difference in the source does not seem to be the primary determinant of the categorical response in the amygdala, because activation of the accessory olfactory bulb appears to be uncategorized. Hamster urine would not be appropriate for these experiments, because it does not carry the same information as urine does in mice and cats (Johnston, 1977). Presumably because hamsters come from arid regions where they must conserve water, they have developed other channels of communication. They produce small volumes of concentrated urine and pay little attention to urine from other hamsters.

The categorical response in the medial amygdala was unchanged in anosmic animals, so it does not depend on input from the olfactory system, suggesting that both conspecific and heterospecific responses result from vomeronasal input. Zinc sulfate-induced anosmia does not destroy all of the olfactory epithelium, but the remaining sensory epithelium is inactivated or blocked by debris for some days and is unable to function for odor detection (Meredith and O'Connell, 1988; McBride et al., 2003).

The MeA is activated by all of the animal stimuli used here and has strong projections to the MeP. Thus, it is in a position to evaluate different spatiotemporal patterns of input from the $\mathrm{AOB}$ and engage excitatory or inhibitory events in the MeP. Preliminary data (not shown) suggest that conspecific stimuli differentially activate the MeP, a region of high expression of steroid receptors (Wood and Newman, 1993). For conspecific stimuli, the $\mathrm{MeP}$ could process information necessary for gender-specific social responses (Lehman and Winans, 1982) but appears to be uninformed about heterospecific stimulation. Thus, the critical discrimination between conspecific (socially relevant) and heterospecific (socially nonrelevant) chemosensory stimuli is more likely a function of the anterior medial amygdala. This simple and perhaps primitive function of the amygdala presages the more complex and perhaps more advanced functions of other amygdala circuits, which can alter the affective-emotional value assigned to stimuli on the basis of conditioned association (Appleton, 2000; Gottfried et al., 2003). The apparent participation of the ICN in the medial amygdala circuit suggests some similarity with the fear-conditioning circuit involving the basolateral and central amygdala (Pare et al., 2003). However, there was no apparent relationship, for any stimuli here, between ICNc activation and that of surrounding amygdaloid nuclei other than MeP, including basolateral and central nuclei, and conditioning is presumably not involved in these responses to first exposure to the stimuli.

\section{References}

Appleton JP (2000) The amygdala, Ed 2. New York: Oxford UP.

Bronson FH (1979) The reproductive ecology of the house mouse. Q Rev Biol 54:265-299.

Coolen LM, Wood RI (1998) Bidirectional connections of the medial amygdaloid nucleus in the Syrian hamster brain: simultaneous anterograde and retrograde tract tracing. J Comp Neurol 399:189-209.

Del Punta K, Puche A, Adams NC, Rodriguez I, Mombaerts P (2002) A divergent pattern of sensory axonal projections is rendered convergent by second-order neurons in the accessory olfactory bulb. Neuron 35:1057-1066.

Dulac C, Torello AT (2003) Molecular detection of pheromone signals in mammals: from genes to behaviour. Nat Rev Neurosci 4:551-562.

Fernandez-Fewell GD, Meredith M (1998) Olfactory contribution to Fos expression during mating in inexperienced male hamsters. Chem Senses 23:257-267.

Gottfried JA, O’DohertyJ, Dolan RJ (2003) Encoding predictive reward value in human amygdala and orbitofrontal cortex. Science 301:1104-1107.

Halpern M, Martinez-Marcos A (2003) Structure and function of the vomeronasal system: an update. Prog Neurobiol 70:245-318.

Hayashi Y, Momiyama A, Takahashi T, Ohishi H, Ogawa-Meguro R, Shigemoto R, Mizuno N, Nakanishi S (1993) Role of a metabotropic glutamate receptor in synaptic modulation in the accessory olfactory bulb. Nature 366:687-690.

Jacob S, McClintock MK, Zelano B, Ober C (2002) Paternally inherited HLA alleles are associated with women's choice of male odor. Nat Genet 30:175-179.

Johnston R, Brenner D (1982) Species specificity of scent marking in hamsters. Behav Neural Biol 35:46-55.

Johnston RE (1977) Sex pheromones in golden hamsters. In: Chemical signals in vertebrates (Muller-Schwarze D, Mozell MM, eds), pp 225-249. New York: Plenum.

Johnston RE (1998) Pheromones, the vomeronasal system, and communication. From hormonal responses to individual recognition. Ann NY Acad Sci 855:333-348.

Kollack-Walker S, Newman SW (1997) Mating-induced expression of c-fos in the male Syrian hamster brain: role of experience, pheromones, and ejaculations. J Neurobiol 32:481-501.

Lehman MN, Winans SS (1982) Vomeronasal and olfactory pathways to the 
amygdala controlling male hamster sexual behavior: autoradiographic and behavioral analyses. Brain Res 240:27-41.

Leinders-Zufall T, Lane AP, Puche AC, Ma W, Novotny MV, Shipley MT, Zufall F (2000) Ultrasensitive pheromone detection by mammalian vomeronasal neurons. Nature 405:792-796.

Lerant A, Kanyicska B, Freeman ME (2001) Nuclear translocation of STAT5 and increased expression of Fos related antigens (FRAs) in hypothalamic dopaminergic neurons after prolactin administration. Brain Res 904:259-269.

Luo M, Fee MS, Katz LC (2003) Encoding pheromonal signals in the accessory olfactory bulb of behaving mice. Science 299:1196-1201.

McBride K, Slotnick B, Margolis FL (2003) Does intranasal application of zinc sulfate produce anosmia in the mouse? An olfactometric and anatomical study. Chem Senses 28:659-670.

Meredith M (1986) Vomeronasal organ removal before sexual experience impairs male hamster mating behavior. Physiol Behav 36:737-743.

Meredith M (1994) Chronic recording of vomeronasal pump activation in awake behaving hamsters. Physiol Behav 56:345-354.

Meredith M (1998) Vomeronasal, olfactory, hormonal convergence in the brain. Cooperation or coincidence? Ann NY Acad Sci 855:349-361.

Meredith M (2001) Human vomeronasal organ function: a critical review of best and worst cases. Chem Senses 26:433-445.

Meredith M, Fewell G (2001) Vomeronasal organ: electrical stimulation activates Fos in mating pathways and in GnRH neurons. Brain Res 922:87-94.

Meredith M, O'Connell RJ (1988) HRP uptake by olfactory and VN recep- tor neurons: use as an indicator of incomplete lesions and relevance for non-volatile chemoreception. Chem Senses 13:487-515.

Morgan JI, Curran T (1991) Stimulus-transcription coupling in the nervous system: involvement of the inducible proto-oncogenes fos and jun. Annu Rev Neurosci 14:421-451.

Murphy MR (1980) Sexual preferences of male hamsters: importance of preweaning and adult experience, vaginal secretion, and olfactory or vomeronasal sensation. Behav Neural Biol 30:323-340.

Nitecka L, Ben-Ari Y (1987) Distribution of GABA-like immunoreactivity in the rat amygdaloid complex. J Comp Neurol 266:45-55.

Nolte CN, Meredith M (1999) Accessory olfactory bulb mGluR2 activation affects pheromone-mediated mating behavior in male Syrian hamsters. Soc Neurosci Abstr 25:55.15.

Pare D, Royer S, Smith Y, Lang EJ (2003) Contextual inhibitory gating of impulse traffic in the intra-amygdaloid network. Ann NY Acad Sci 985:78-91.

Verberne G (1976) Chemocommunication among domestic cats, mediated by the olfactory and vomeronasal senses. II. The relation between the function of Jacobson's organ (vomeronasal organ) and Flehmen behaviour. Z Tierpsychol 42:113-128.

Wood RI, Newman SW (1993) Mating activates androgen receptorcontaining neurons in chemosensory pathways of the male Syrian hamster brain. Brain Res 614:65-77.

Wysocki C, Meredith M (1987) The vomeronasal system. In: Neurobiology of taste and smell (Finger TH, Silver WL, eds), pp 125-150. New York: Wiley. 